\title{
Potential effects of valproate and oxcarbazepine on growth velocity and bone metabolism in epileptic children- a medical center experience
}

Chien-Ming Lin ${ }^{1,2}$, Hueng-Chuen Fan ${ }^{1,3}$, Tsu-Yi Chao ${ }^{2,4,5}$, Der-Ming Chu', Chi-Chieh Lai ${ }^{1}$, Chih-Chien Wang ${ }^{1}$ and Shyi-Jou Chen ${ }^{1 *}$

\begin{abstract}
Background: Children with longstanding use of antiepileptic drugs (AEDs) are susceptible to developing low bone mineral density and an increased fracture risk. However, the literature regarding the effects of AEDs on growth in epileptic children is limited. The aim of this study was to investigate the potential effects of valproate (VPA) and/or oxcarbazepine (OXC) therapy on growth velocity and bone metabolism.

Methods: Seventy-three ambulatory children (40 boys and 33 girls) with epilepsy, aged between 1 and 18 years (mean age $9.8 \pm 4.1$ years), were evaluated for growth velocity before and for 1 year after VPA and/or OXC treatment. The bone resorption marker serum tartrate-resistant acid phosphatase $5 b$ (TRAcP5b) and the bone formation marker serum bone-specific alkaline phosphatase (BAP) were measured post-AEDs therapy for 1 year.

Results: The difference in growth velocity $(\Delta \mathrm{Ht})$ and body weight change $(\Delta \mathrm{Wt})$ between pre- and post-AEDs treatment were $-1.0 \pm 2.8 \mathrm{~cm} /$ year $(P<0.05)$ and $0.1 \pm 3.9 \mathrm{~kg} /$ year $(P=0.84)$, respectively. The study population had serum TRACP5b-SDS of $-1.6 \pm 1.2$ and BAP-SDS of $1.7 \pm 3.7$ compared with sex- and age-matched healthy children. Significant correlation between serum TRAcP $5 b$ and BAP activities was noted $(r=0.60, p<0.001)$. There was a positive correlation between growth velocity and serum TRACP 5b activity after AED treatment $(r=0.42, p<0.01)$. No correlation was found between $\Delta \mathrm{Ht}, \Delta \mathrm{Wt}$, serum TRAcP $5 \mathrm{~b}, \mathrm{BAP}$ activity and types of AEDs.

Conclusion: Growth velocity was significantly decreased in epileptic children after 1 year of VPA and/or OXC treatment. The effect of VPA and/or OXC therapy on dysregulation of bone metabolism might play a crucial role in physical growth.
\end{abstract}

Keywords: Antiepileptic drugs, Growth velocity, Bone metabolism, Tartrate-resistant acid phosphatase 5b, Bonespecific alkaline phosphatase

\section{Background}

Antiepileptic drugs (AEDs) have been shown to be associated with a lowering of bone mineral density (BMD) in childhood and adolescence [1-3], which are critical periods for bone mineralization [4]. The underlying pathophysiological mechanisms of AEDs on bone metabolism

\footnotetext{
* Correspondence: pedneuchen@hotmail.com

'Department of Pediatrics, Tri-Service General Hospital, National Defense Medical Center, No.325, Cheng-Kung Road, Section 2, Nei-Hu, Taipei 114, Taiwan

Full list of author information is available at the end of the article
}

are poorly understood but are probably multifactorial, and include inducing cytochrome p450 enzyme activities [5], increased bone turnover [6], inhibition of osteocalcin [7], and increased urinary loss of calcium and phosphorus.

Along with decreased BMD, the disturbance of physical growth during AEDs treatment is also of great concern to physicians. It has been reported that long-term valproate (VPA) and lamotrigine (LTG) therapy, particularly when combined, is associated with short stature [8]. However, the alteration in growth is postulated to be mediated through immobility, not by the AEDs therapy 
itself [8]. In addition to the investigations into static height only [8], Rattya J et al. conducted a longitudinal growth analysis to evaluate the effect of VPA, carbamazepine (CBZ), or oxcarbazepine (OXC) therapy on physical growth [9]. Of note, AEDs treatment did not affect linear growth in girls with epilepsy [9]. Taken together, findings regarding the adverse effects of AEDs therapy on growth stature are still inconsistent $[8,9]$.

Furthermore, the literature regarding the influence of AEDs on dynamic height (growth velocity) in epileptic children is limited, and the effects of AEDs on growth via disregulation of bone mineralization are not fully known. Therefore, we conducted a cross-sectional cohort study to evaluate growth velocity and bone turnover markers in growing Taiwanese children with epilepsy to identify the potential effects of VPA and $\mathrm{OXC}$ therapy on decreased growth velocity and disruption of bone metabolism.

\section{Methods}

\section{Participants and study design}

Seventy-three ambulatory outpatients with epilepsy aged 1 to 18 years who visited the Department of Pediatrics of Tri-Service General Hospital in Taiwan were enrolled. Based on the International League Against Epilepsy classification [10], all patients were definitively diagnosed with epilepsy or epileptic syndrome and were taking VPA $(n=23)$, OXC $(n=29)$, or VPA combined with OXC $(n=21)$. All of them were free of seizure for longer than 6 months after AEDs treatment and had an active daily life. Exclusion criteria were acute or chronic disease affecting bone metabolism, significant disabilities such as mental or motor retardation, endocrinologic diseases, taking medications other than AEDs, or any other comorbidity, such as muscular dystrophy, neurological conditions, or recent osteomyelitis. Legal guardians or parents of subjects aged less than 7 years signed an informed consent. Both subjects and their legal guardians or parents had to sign a combination assent/parental permission form written simply and at a comprehensive level appropriate for children between ages 7 to 18 . The Ethics Review Committee of Tri-Service General Hospital approved this study.

\section{Anthropometry}

Because growth velocity varies at different stages of life, participants were classified into three subgroups according to pubertal age: pre-puberty (boy $1-8$ years, girl 17 years), puberty (boy 9-14 years, girl 8-13 years), and post-puberty (boy 15-18 years, girl 14-18 years). Retrospective growth data were gathered only from the records at Tri-Service General Hospital, with an accuracy of $0.5 \mathrm{~cm}$ for height and $0.1 \mathrm{~kg}$ for weight. Height was measured to the nearest $1.0 \mathrm{~mm}$ with the same
Harpenden wall-mounted stadiometer and weight to the nearest $0.1 \mathrm{~kg}$ on a calibrated weight scale at the clinical examination. The difference in growth velocity and body weight change before and for 1 year after AEDs treatment was expressed as $\Delta \mathrm{Ht}$ and $\Delta \mathrm{Wt}$, respectively.

\section{Blood sampling}

Upon entry, a blood sample was drawn at 8:00 AM after an overnight fast for the evaluation of plasma aspartate aminotransferase (AST), alanine aminotransferase (ALT), blood urea nitrogen (BUN), creatinine (Cre), alkaline phosphatase (ALP), total calcium (Ca), free $\mathrm{Ca}$, phosphorus (P), and blood hemoglobin (Hgb). Another blood sample was taken for two bone turnover markers, the serum bone resorption marker TRAcP $5 \mathrm{~b}[11,12]$ and the bone formation marker BAP activities, after AEDs therapy for 12 months. Serum TRAcP 5b was assessed by a solid phase, immunofixed, enzyme activity assay as described previously [13] with an intra- and inter-assay CV of $5.1 \%$ and $3.9 \%$, respectively. BAP activity was measured using a commercially available quantitative ELISA kit (Metra BAP EIA kit, Quidel Corp., San Diego, CA) in which serum BAP was immobilized by a specific antibody and its activity measured using 4-nitrophenyl phosphate as the substrate.

\section{Statistical analyses}

Basic data were calculated as mean values with standard deviation. Anthropometry data, serum TRAcP 5b and BAP activities were compared with age- and sexspecific Taiwanese reference values and presented as standard deviation scores $(\mathrm{SDS}=[x$-mean $] / \mathrm{SD})[14,15]$. The statistical analysis was performed by means of the Student's $t$ test (two-tailed) and one-way analysis of variance (ANOVA) for comparison of continuous variables. Pearson's correlation analysis was used to estimate the relationships between serum TRAcP 5b, BAP activities, and growth velocity after AEDs treatment. A $p$-value $<0.05$ was considered significant.

\section{Results}

Basic demographic and biochemical data of epileptic children at baseline and 1 year post-AEDs treatment are shown in Table 1 . The 73 patients (40 boys and 33 girls) had a mean age ( \pm SD) of $9.8 \pm 4.1$ years, heightSDS of $-0.2 \pm 1.3$, weight-SDS of $0.5 \pm 1.8$, and body mass index-SDS of $1.1 \pm 2.6$ [14]. The baseline serum levels of AST/ALT, BUN/Cre, ALP, total Ca, free Ca, P and blood $\mathrm{Hgb}$ were all within normal ranges. The mean TRAcP 5b activity was $4.8 \pm 1.7 \mu \mathrm{mol} / \mathrm{L} / \mathrm{min}$ and the mean BAP activity was $144.4 \pm 73.2 \mu \mathrm{mol} / \mathrm{L} / \mathrm{min}$ after AEDs treatment for 1 year. Compared with the sexand age-matched healthy Taiwanese children, TRAcP5bSDS was $-1.6 \pm 1.2$ and BAP-SDS was $1.7 \pm 3.7$ [15]. 
Table 1 Demographic and biochemical data of epileptic children at baseline and post-AED treatment ${ }^{a}$

\begin{tabular}{|c|c|}
\hline Baseline & \\
\hline \multicolumn{2}{|l|}{ Overall gender $(\mathrm{N})$} \\
\hline Male/Female & $40 / 33$ \\
\hline Total & 73 \\
\hline Age (yrs) & $9.8 \pm 4.1$ \\
\hline \multicolumn{2}{|l|}{ Type of AEDs (N) } \\
\hline Valproate & 23 \\
\hline Oxcarbazepine & 29 \\
\hline Valproate + Oxcarbazepine & 21 \\
\hline \multicolumn{2}{|l|}{ Growth evaluation } \\
\hline Height (cm) & $133.5 \pm 21.5$ \\
\hline Height-SDS & $-0.2 \pm 1.3$ \\
\hline Weight (kg) & $35.0 \pm 17.0$ \\
\hline Weight-SDS & $0.5 \pm 1.8$ \\
\hline Body mass index-SDS & $1.1 \pm 2.6$ \\
\hline \multicolumn{2}{|l|}{ Laboratory studies } \\
\hline Aspartate aminotransferase $(U / L)$ & $27.6 \pm 7.5$ \\
\hline Alanine aminotransferase $(\mathrm{U} / \mathrm{L})$ & $17.1 \pm 7.8$ \\
\hline Blood urea nitrogen (mg/dL) & $13.0 \pm 1.2$ \\
\hline Creatinine (mg/dL) & $0.5 \pm 0.2$ \\
\hline Alkaline phosphatase (U/L) & $226.8 \pm 85.0$ \\
\hline Total calcium (mg/dL) & $9.5 \pm 0.4$ \\
\hline Free calcium (mg/dL) & $4.9 \pm 0.3$ \\
\hline Phosphorous (mg/dL) & $4.7 \pm 0.3$ \\
\hline Hemoglobin (g/dL) & $13.0 \pm 1.2$ \\
\hline \multicolumn{2}{|l|}{ Post-treatment } \\
\hline \multicolumn{2}{|l|}{ Laboratory studies } \\
\hline Serum TRAcP5b (umol/L/min) & $4.8 \pm 1.7$ \\
\hline TRACP5b-SDS & $-1.6 \pm 1.2$ \\
\hline Serum BAP (umol/L/min) & $144.4 \pm 73.2$ \\
\hline BAP-SDS & $1.7 \pm 3.7$ \\
\hline Total calcium (mg/dL) & $9.2 \pm 0.3$ \\
\hline Free calcium (mg/dL) & $4.7 \pm 0.3$ \\
\hline
\end{tabular}

${ }^{a}$ Data presented as mean \pm SD
Growth velocity post-AEDs treatment had a decreasing trend in all three subgroups $(\Delta \mathrm{Ht}$ : prepuberty $-1.4 \pm 3.3 \mathrm{~cm} /$ year; puberty $-0.7 \pm 2.6 \mathrm{~cm} /$ year; post-puberty $-0.7 \pm 1.8 \mathrm{~cm} /$ year) (Table 2 ). Overall, the difference in growth velocity between pre- and postAEDs treatment was $-1.0 \pm 2.8 \mathrm{~cm} /$ year $(P<0.05)$. The differences in body weight change between pre- and post-AEDs treatment were $1.2 \pm 3.0 \mathrm{~kg} /$ year in pre-puberty and $-3.3 \pm 6.6 \mathrm{~kg} /$ year in post-puberty, respectively. Among all participants, there was no significant difference in body weight change between pre- and post-AEDs treatment $(P=0.84)$.

There was a significant correlation between serum TRAcP $5 \mathrm{~b}$ and BAP activity after AED treatment $(r=$ $0.60, p<0.001$ ) (data not shown). In Table 3, a positive correlation between growth velocity after AEDs treatment and serum TRAcP $5 \mathrm{~b}$ activity was noted in the post-pubertal group $(r=0.93, p<0.01)$ and in all patients $(r=0.42, p<0.01)$. No correlation was found between $\Delta \mathrm{Ht}, \Delta \mathrm{Wt}$, serum TRAcP $5 \mathrm{~b}, \mathrm{BAP}$ activity and types of AEDs (data not shown).

\section{Discussion}

Our findings of a decrease in the bone resorption marker TRAcP 5b-SDS $(-1.6 \pm 1.2)$, an increase in the bone formation marker BAP-SDS (1.7 \pm 3.7$)$, and a significant correlation between serum TRAcP $5 \mathrm{~b}$ and BAP activities $(r=0.60, p<0.001)$ might be indirect evidence of increased bone turnover. In terms of body height, a decreased growth velocity after VPA and/or OXC therapy for 1 year $(-1.0 \pm 2.8 \mathrm{~cm} /$ year, $p<0.05)$ and a significantly positive correlation between growth velocity and serum TRAcP 5b activity after AEDs therapy $(r=0.42$, $p<0.01)$ were observed in all subjects. These interesting findings might partly explain the crucial role of the uncoupling of bone metabolism in decelerated physical growth in growing children with short-term VPA and/ or OXC treatment. To our knowledge, the present study is the first report to delineate the relationship between decreased growth velocity and the short-term use of VPA and/or OXC therapy in a representative Taiwan population-based sample. However, a wellcontrolled longitudinal study with more patients and

Table 2 Growth velocity and body weight change of epileptic children before and after AEDs treatment

\begin{tabular}{|c|c|c|c|c|c|c|c|c|c|c|}
\hline \multirow[t]{2}{*}{ Patients } & \multicolumn{2}{|l|}{ No. } & \multicolumn{4}{|c|}{ Growth velocity (cm/yr) } & \multicolumn{4}{|c|}{ Body weight change (kg/yr) } \\
\hline & $\mathrm{M} / \mathrm{F}$ & Total & Pre-AEDs & Post-AEDs & $\Delta \mathrm{Ht}^{\mathrm{a}}$ & $p$-value* & Pre-AEDs & Post-AEDs & $\Delta W t^{b}$ & $p$-value ${ }^{*}$ \\
\hline Pre-puberty & $19 / 9$ & 28 & $6.7 \pm 3.4$ & $5.3 \pm 1.3$ & $-1.4 \pm 3.3$ & 0.13 & $1.9 \pm 1.8$ & $2.7 \pm 2.0$ & $1.2 \pm 3.0$ & 0.33 \\
\hline Puberty & $13 / 17$ & 30 & $6.5 \pm 3.4$ & $5.8 \pm 2.0$ & $-0.7 \pm 2.6$ & 0.30 & $3.9 \pm 2.8$ & $4.7 \pm 2.7$ & $0.5 \pm 3.0$ & 0.29 \\
\hline Post-puberty & $8 / 7$ & 15 & $3.7 \pm 2.8$ & $3.0 \pm 1.9$ & $-0.7 \pm 1.8$ & 0.35 & $4.3 \pm 7.4$ & $1.0 \pm 2.3$ & $-3.3 \pm 6.6$ & 0.27 \\
\hline Overall & $40 / 33$ & 73 & $6.1 \pm 3.4$ & $5.2 \pm 2.0$ & $-1.0 \pm 2.8$ & 0.04 & $3.2 \pm 3.6$ & $3.4 \pm 2.7$ & $0.1 \pm 3.9$ & 0.84 \\
\hline
\end{tabular}

Data presented as mean $\pm \mathrm{SD} ;{ }^{\text {a }}$ The difference in growth velocity between pre- and post-AEDs treatment; ${ }^{\mathrm{b}}$ The difference in body weight change between preand post-AEDs treatment; ${ }^{*} P$-value between pre- and post-AEDs treatment 
Table 3 Correlation of coefficient between growth velocity after AEDs treatment and bone turnover markers

\begin{tabular}{lllll}
\hline & \multicolumn{4}{l}{ Growth velocity after AEDs treatment } \\
\cline { 2 - 5 } & Pre-puberty & Puberty & Post-puberty & Overall \\
\hline Serum TRACP5b & 0.07 & 0.23 & $0.93^{*}$ & $0.42^{*}$ \\
Serum BAP & 0.04 & 0.01 & 0.49 & 0.22 \\
\hline${ }^{*} P<0.01$ & & & &
\end{tabular}

evaluation of more bone turnover markers is needed to confirm our hypothesis.

Both enzyme-inducing and non-enzyme inducing AEDs can cause abnormalities in bone metabolism [16-19]. The negative influence of AEDs on BMD is complex and not understood completely, but several lines of evidence suggest a role for the induction of cytochrome p450 enzyme activities, which accelerate vitamin D hydroxylation to inactive forms [5], increased bone turnover [6], inhibition of osteocalcin [7], and decreased intestinal transport of calcium [20]. Besides decreased BMD, the influence of AEDs treatment on physical growth is of great concern in clinical practice nowadays. However, the literature regarding the adverse effect of AEDs therapy on growth stature is very limited and the results are inconsistent $[8,9]$. Guo et al. reported long-term VPA and LTG therapy, particularly when combined, is associated with short stature, low $\mathrm{BMD}$, and reduced bone formation; but these alterations are thought to be mediated primarily through reduced physical activity rather than though a direct link to AEDs therapy [8]. In contrast to the investigation of static height alone [8], our study found epileptic children with normal daily activity had decreased dynamic growth patterns associated with increased bone turnover after short-term VPA and/or OXC treatment. Although Rattya et al. reported VPA, CBZ, or OXC seem not to have an adverse effect on linear growth in girls with epilepsy, VPA-related weight gain could be observed in that series [9]. As illustrated, weight gain induced advanced bone age resulting in rapid growth may partially mask the true effect of VAP on growth in that series. On the contrary, our three study subgroups all had a trend toward a decrease in growth velocity, no matter whether body weight increased or not. There was a lack of obvious weight gain in our study, which is typically associated with a protective effect on bone mineralization [21], so we supposed that the decelerated growth velocity might not be attributed to body weight change, but to AEDs treatment itself. Nevertheless, the effect of the interaction between AEDs therapy and genetic, hormonal, and environmental factors on physical growth in Taiwanese epileptic children still should be considered.

In adults and children, VPA therapy is associated with weight gain, high body fat, and hyperleptinaemia $[9,22]$. Thus, it is speculated that VPA could affect bone mass accrual and/or turnover indirectly through weight gain and the associated hyperleptinaemia [19]. However, the AEDs-induced weight gain was only observed in patients taking VPA, not in those treated with OXC [9]. In contrast to previous reports $[9,22]$, the differences in body weight change between pre- and post-VPA and/or OXC treatment did not alter significantly in the present study $(p=0.84)$. Furthermore, our study found no correlation between $\Delta \mathrm{Wt}$ and usage of types of AEDs (data not shown). We supposed that the effect of VPA on weight gain in all participants might be masked by decreased body weight gain in the post-pubertal subgroup, in consideration of the elevated self-image and sense of selfesteem, as well as the lack of body weight change with OXC therapy [9]. Of importance, the weight gain in patients treated with VPA was slow but progressive [9], which may subsequently increase weight-bearing for bone remodeling, BMD, and linear growth. Therefore, the possibility of weight gain in epileptic patients should be monitored during long-term VPA treatment to elucidate its true effect on bone growth.

Serum vitamin D levels were not measured in our study. Vitamin D deficiency in participants with epilepsy was reported to be common in some, but not all, earlier studies [19, 23-25]. These discrepant results may be attributed to whether AEDs, with their cytochrome P450 enzyme inducers, which increase vitamin D metabolism, were used or not. On the other hand, confounding factors such as AEDs polytherapy and immobility may also contribute to vitamin D deficiency/insufficiency in epileptic patients $[8,26]$. In contrast to strong enzyme inducers such as CBZ, non-enzyme-inducing (VPA) and minimal enzyme-inducing (OXC) agents were used by our participants. All subjects in the present study were ambulatory and normally physically active. Furthermore, Taiwan is a subtropical Asian country and so sunlight exposure was also adequate. Herein, disruption to bone metabolism related to vitamin D deficiency seemed not to be an important contributor to decreased growth velocity in our series.

Nevertheless, our study has some limitations. Firstly, there was a lack of X-ray image to investigate the relationship between BMD, bone age study and growth velocity. However, due to the fear of high-level exposure to radiation, the risk-benefit analysis did not justify performing BMD measurements in present study, particularly from a parental point of view. Furthermore, findings regarding the effect of AEDs on BMD are still conflicting [23, 24, 26-28]. Secondly, the functions of insulin like growth factor-I (IGF-I) were potentially to increase markers of osteoblastic activity and reduce bone resorption [29]; and serum IGF-binding proteins-2 (IGFBP-2) levels was associated with low BMD and high bone resorption markers [30,31]. However, the growth 
hormone (GH)/IGF axis, which is essential to maintain homeostasis of bone turnover, was not evaluated in our study [32]. Finally, our patient sample size was small and was not equally distributed across age subgroups, which might explain why the differences in growth velocity between pre- and post-AEDs treatment were significant overall, but not in each subgroup. Therefore, a largerscale prospective study in consideration of IGF-I, IGFBP-2, bone turnover biomarkers, and growth velocity is necessary to clarify our hypothesis of AEDs on growth and bone metabolism.

\section{Conclusion}

Our findings suggest that 1-year survey of VPA and/or OXC therapy among growing epileptic children might be associated with decreased growth velocity. This alteration seems to be mediated through the effect of AEDs on dysregulation of bone metabolism. To monitor growth velocity and bone metabolism, a larger-scale, long-term prospective study considering weight gain effect of AEDs, genetic, hormonal, and nutritional factors is needed to elucidate the true effects of AEDs itself on physical growth.

\section{Availability of data and materials}

The datasets supporting the conclusions of this article is included within the manuscript and additional file 1.

\section{Additional file}

Additional file 1: The orginal data of the study. (XLSX $19 \mathrm{~kb})$

\section{Abbreviations}

AEDs: antiepileptic drugs; BAP: bone-specific alkaline phosphatase; BMD: bone mineral density; CBZ: carbamazepine; LTG: lamotrigine; OXC: oxcarbazepine; TRACP5b: tartrate-resistant acid phosphatase 5b; VPA: valproate.

\section{Competing interests}

The authors declare that they have no competing interests.

\section{Authors' contributions}

CML contributed to initial data analysis and interpretation, drafted the initial manuscript, and approved the final manuscript as submitted. HCF, TYC, DMC, $\mathrm{CCL}$, and $\mathrm{CCW}$ designed the intervention and decided upon the data collection methods. HCF and TYC were also responsible for statistical analysis. SJC conceptualized and designed the study, supervised all aspects of the study, critically reviewed and revised the manuscript, and approved the final manuscript as submitted. All authors read and approved the final manuscript.

\section{Authors' information}

Not applicable

\section{Acknowledgements}

We thank Su-Hui Li and Shin-Yi Liou from the Division of Hematology/ Oncology, Department of Medicine, Tri-Service General Hospital, for their technical assistance.

\section{Funding source}

This work was funded by research grants from the Tri-Service General Hospital (TSGH-C103-019 and TSGH-C104-024) and National Science Council (NSC102-2314-B-016-019-MY3), Taiwan.

\section{Author details}

'Department of Pediatrics, Tri-Service General Hospital, National Defense Medical Center, No.325, Cheng-Kung Road, Section 2, Nei-Hu, Taipei 114, Taiwan. ${ }^{2}$ Graduate Institute of Medical Sciences, National Defense Medical Center, No.161, Cheng-Kung Road, Section 6, Nei-Hu, Taipei 114, Taiwan. ${ }^{3}$ Department of Pediatrics, Tungs' Taichung MetroHarbor Hospital, No.699, Section 1, Zhongqi Road, Wuqi Township, Taichung 435, Taiwan. ${ }^{4}$ Division of Hematology/Oncology, Department of Internal Medicine, Taipei Medical University, Shuang Ho Hospital, No.291, Zhongzheng Road, Zhonghe District, New Taipei City 235, Taiwan. ${ }^{5}$ Graduate Institute of Clinical Medicine, College of Medicine, Taipei Medical University, No.252, Wu Hsing Street, Taipei 110, Taiwan.

Received: 6 April 2014 Accepted: 21 April 2016

Published online: 03 May 2016

\section{References}

1. Pack AM, Gidal B, Vazquez B. Bone disease associated with antiepileptic drugs. Cleve Clin J Med. 2004;71 Suppl 2:S42-8.

2. Chou IJ, Lin KL, Wang HS, Wang CJ. Evaluation of bone mineral density in children receiving carbamazepine or valproate monotherapy. Acta Paediatr Taiwan. 2007:48(6):317-22.

3. Phabphal K, Limapichat K, Sathirapanya P, Setthawatcharawanich S, Leelawattana R, Thammakumpee $\mathrm{N}$, et al. Bone mineral density following long-term use of antiepileptic drugs in a tropical Asian country. Epileptic Disord. 2008;10(3):213-8.

4. Baroncelli GI, Bertelloni S, Sodini F, Saggese G. Osteoporosis in children and adolescents: etiology and management. Paediatr Drugs. 2005;7(5):295-323.

5. Ecevit C, Aydoğan A, Kavakli T, Altinöz S. Effect of carbamazepine and valproate on bone mineral density. Pediatr Neurol. 2004;31(4):279-82.

6. Telci A, Cakatay U, Kurt BB, Kayali R, Sivas A, Akcay T, et al. Changes in bone turnover and deoxypyridinoline levels in epileptic patients. Clin Chem Lab Med. 2000;38(1):47-50.

7. Onodera K, Takahashi A, Mayanagi H, Wakabayashi H, Kamei J, Shinoda H. Phenytoin-induced bone loss and its prevention with alfacalcidol or calcitriol in growing rats. Calcif Tissue Int. 2001;69(2):109-16.

8. Guo $C Y$, Ronen $\mathrm{GM}$, Atkinson SA. Long-term valproate and lamotrigine treatment may be a marker for reduced growth and bone mass in children with epilepsy. Epilepsia. 2001;42(9):1141-7.

9. Rattya J, Vainionpaa L, Knip M, Lanning P, Isojarvi JI. The effects of valproate, carbamazepine, and oxcarbazepine on growth and sexual maturation in girls with epilepsy. Pediatrics. 1999;103(3):588-93.

10. Engel Jr J, International League Against Epilepsy (ILAE). A proposed diagnostic scheme for people with epileptic seizures and with epilepsy: report of the ILAE Task Force on Classification and Terminology. Epilepsia. 2001;42(6):796-803.

11. Halleen JM, Alatalo SL, Suominen H, Cheng S, Janckila AJ, Vaananen HK. Tartrate-resistant acid phosphatase $5 \mathrm{~b}$ : a novel serum marker of bone resorption. J Bone Miner Res. 2000;15(7):1337-45.

12. Janckila AJ, Takahashi K, Sun SZ, Yam LT. Tartrate-resistant acid phosphatase isoform 5b as serum marker for osteoclastic activity. Clin Chem. 2001;47(1): $74-80$.

13. Chao TY, Ho CL, Lee SH, Chen MM, Janckila A, Yam LT. Tartrate-resistant acid phosphatase $5 \mathrm{~b}$ as a serum marker of bone metastasis in breast cancer patients. J Biomed Sci. 2004;11(4):511-6.

14. Chen W, Chang MH. New growth charts for Taiwanese children and adolescents based on World Health Organization standards and healthrelated physical fitness. Pediatr Neonatol. 2010;51(2):69-79.

15. Chen CJ, Chao TY, Janckila AJ, Cheng SN, Ku CH, Chu DM. Evaluation of the activity of tartrate-resistant acid phosphatase isoform $5 b$ in normal Chinese children-a novel marker for bone growth. J Pediatr Endocrinol Metab. 2005; 18(1):55-62.

16. Andress DL, Ozuna J, Tirschwell D, Grande L, Johnson M, Jacobson AF, et al. Antiepileptic drug-induced bone loss in young male patients who have seizures. Arch Neurol. 2002;59(5):781-6.

17. Boluk A, Guzelipek M, Savli H, Temel I, Ozişik HI, Kaygusuz A. The effect of valproate on bone mineral density in adult epileptic patients. Pharmacol Res. 2004;50(1):93-7.

18. Pack AM, Olarte LS, Morrell MJ, Flaster E, Resor SR, Shane E. Bone mineral density in an outpatient population receiving enzyme-inducing antiepileptic drugs. Epilepsy Behav. 2003;4(2):169-74. 
19. Rauchenzauner M, Griesmacher A, Tatarczyk T, Haberlandt E, Strasak A, Zimmerhackl LB, et al. Chronic antiepileptic monotherapy, bone metabolism, and body composition in non-institutionalized children. Dev Med Child Neurol. 2010;52(3):283-8.

20. Välimäki MJ, Tiihonen MLK, Tähtelä RKM, Lamberg-Allardt C, Mäkelä P, Tunninen R. Bone mineral density measured by dual-energy $x$-ray absorptiometry and novel markers of bone formation and resorption in patients on antiepileptic drugs. J Bone Miner Res. 1994;9(5):631-7.

21. Fitzpatrick LA. Pathophysiology of bone loss in patients receiving anticonvulsant therapy. Epilepsy Behav. 2004;5 Suppl 2:S3-15.

22. Rauchenzauner M, Haberlandt E, Scholl-Burgi S, Karall D, Schoenherr E, Tatarczyk T, et al. Effect of valproic acid treatment on body composition, leptin and the soluble leptin receptor in epileptic children. Epilepsy Res. 2008;80(2-3):142-9.

23. Farhat G, Yamout B, Mikati MA, Demirjian S, Sawaya R, El-Hajj Fuleihan G Effect of antiepileptic drugs on bone density in ambulatory patients. Neurology. 2002;58(9):1348-53.

24. Rieger-Wettengl G, Tutlewski B, Stabrey A, Rauch F, Herkenrath P, SchauseilZipf U, et al. Analysis of the musculoskeletal system in children and adolescents receiving anticonvulsant monotherapy with valproic acid or carbamazepine. Pediatrics. 2001;108(6):E107.

25. Verrotti A, Greco R, Latini G, Morgese G, Chiarelli F. Increased bone turnover in prepubertal, pubertal, and postpubertal patients receiving carbamazepine. Epilepsia. 2002;43(12):1488-92.

26. El-Hajj Fuleihan G, Dib L, Yamout B, Sawaya R, Mikati MA. Predictors of bone density in ambulatory patients on antiepileptic drugs. Bone. 2008:43(1):149-55.

27. Kim SH, Lee JW, Choi KG, Chung HW, Lee HW. A 6-month longitudinal study of bone mineral density with antiepileptic drug monotherapy. Epilepsy Behav. 2007;10(2):291-5.

28. Akin R, Okutan V, Sarici U, Altunbas A, Gökçay E. Evaluation of bone mineral density in children receiving antiepileptic drugs. Pediatr Neurol. 1998;19(2): 129-31.

29. Guerra-Menéndez L, Sádaba MC, Puche JE, Lavandera JL, de Castro LF, de Gortázar AR, et al. IGF-I increases markers of osteoblastic activity and reduces bone resorption via osteoprotegerin and RANK-ligand. J Transl Med. 2013;11:271.

30. Amin S, Riggs BL, Atkinson EJ, Oberg AL, Melton 3rd LJ, Khosla S. A potentially deleterious role of IGFBP-2 on bone density in aging men and women. J Bone Miner Res. 2004;19(7):1075-83.

31. Amin S, Riggs BL, Melton 3rd LJ, Achenbach SJ, Atkinson EJ, Khosla S. High serum IGFBP-2 is predictive of increased bone turnover in aging men and women. J Bone Miner Res. 2007;22(6):799-807.

32. Yamamoto M, Sugimoto T. Effects of growth hormone replacement therapy on bone metabolism. Clin Calcium. 2014;24(6):903-9.

\section{Submit your next manuscript to BioMed Central and we will help you at every step:}

- We accept pre-submission inquiries

- Our selector tool helps you to find the most relevant journal

- We provide round the clock customer support

- Convenient online submission

- Thorough peer review

- Inclusion in PubMed and all major indexing services

- Maximum visibility for your research

Submit your manuscript at www.biomedcentral.com/submit
Biomed Central 